POS $\quad \begin{aligned} & \text { PROCEEDINGS } \\ & \text { OF SCIENCE }\end{aligned}$

\title{
One-Flavor Algorithms for Simulation of Lattice QCD with Domain-Wall Fermion: EOFA versus RHMC
}

Yu-Chih Chen*1, Ting-Wai Chiu ${ }^{1,2}$ (TWQCD Collaboration)

${ }^{1}$ Physics Department, National Taiwan University, Taipei 10617, Taiwan

${ }^{2}$ Center for Quantum Science and Engineering, National Taiwan University,

Taipei 10617, Taiwan

We compare the performances of the exact one-flavor algorithm (EOFA) and the rational hybrid Monte Carlo algorithm (RHMC), for dynamical simulations of lattice QCD with domain-wall fermion.

2013 International Workshop on Computational Science and Engineering,

14-17 October 2013,

National Taiwan University, Taipei, Taiwan

${ }^{*}$ Speaker. 


\section{Introduction}

Recently, an exact pseudofermion action for hybrid Monte Carlo simulation (HMC) of lattice QCD with one-flavor of domain-wall fermion (DWF) has been derived, with the effective 4-dimensional Dirac operator equal to the optimal rational approximation of the overalp Dirac operator with kernel $H=c H_{w}\left(1+d \gamma_{5} H_{w}\right)^{-1}$, where $c$ and $d$ are constants, and $H_{w}$ is the standard Wilson-Dirac operator plus a negative parameter $-m_{0}\left(0<m_{0}<2\right)$ [1]. Since the action is exact without taking square root, it does not require a large memory space to compute the fermion force, unlike the widely used rational hybrid Monte Carlo algorithm (RHMC) [2]. In the following, we refer the HMC with the exact one-flavor pseudofermion action as the exact one-flavor algorithm (EOFA). Obviously, the memory-saving feature of EOFA is crucial for large-scale simulations of lattice QCD on any platforms. This is especially true for GPUs, since each GPU has enormous floating-point computing power but limited device memory. For example, using EOFA, two GPUs (each of 6 GB device momory, e.g., Nvidia GTX-TITAN) working together is capable to simulate lattice QCD with $(u, d, s, c)$ DWF quarks on the $32^{3} \times 64 \times 16$ lattice, while this is infeasible for RHMC. About the computational efficiency of EOFA, our studies in Ref. [1] suggest that EOFA is compatible with RHMC. However, in Ref. [1], a salient feature of EOFA has not been exploited. Namely, $\phi_{1}$ and $\phi_{2}$ [see Eq. (23) of Ref. [1]] can be updated at two different time scales, since the fermion force of $\phi_{1}$ is much smaller than that of $\phi_{2}$. Now, applying the multiple-time scale method to $\phi_{1}$ and $\phi_{2}$, we find that EOFA is more efficient than RHMC for all variants of DWF. In this paper, we demonstrate that this is the case for the conventional DWF with kernel $H=2 c H_{w}\left(2+\gamma_{5} H_{w}\right)^{-1}$ (i.e, $d=1 / 2$, and $\omega=1$ ), and the optimal DWF [3] with kernel $H=H_{w}$ (i.e., $c=1, d=0$, and the reflection-symmetric $\omega$ ), and the tests are performed for $N_{f}=1$ and $N_{f}=(2+1)$ QCD respectively.

Since the details of EOFA have been given in Ref. [1], we do not repeat them here. In the following, we outline how we implement RHMC in our tests. The pseudofermion action for RHMC of $N_{f}=1 \mathrm{DWF}$ can be written as

$$
S_{p f}^{N_{f}=1}=\phi^{\dagger}\left(C_{1}^{\dagger} C_{1}\right)^{1 / 4}\left(C C^{\dagger}\right)^{-1 / 2}\left(C_{1}^{\dagger} C_{1}\right)^{1 / 4} \phi,
$$

where $C$ is defined as [4],

$$
\begin{aligned}
C(m) & \equiv I-M_{5}(m) D_{w}^{o e} M_{5}(m) D_{w}^{e o}, C_{1} \equiv C(1) \\
\left(D_{w}^{e o(o e)}\right)_{x, y} & \equiv \frac{1}{2}\left[\left(\gamma_{\mu}-1\right) U_{\mu}(x) \delta_{y, x+\mu}-\left(\gamma_{\mu}+1\right) U_{\mu}^{\dagger}(y) \delta_{y, x-\mu}\right], \\
M_{5}(m) & \equiv\left[4-m_{0}+P_{+} M_{+}(m)+P_{-} M_{-}(m)\right]^{-1},
\end{aligned}
$$

and the number of poles in the optimal rational approximation of $\left(C C^{\dagger}\right)^{-1 / 2}$ and $\left(C_{1}^{\dagger} C_{1}\right)^{1 / 4}$ is $N_{p}$. The pseudofermion field $\phi$ is generated from the Gaussian noise field $\eta$ as follows.

$$
\phi=\frac{1}{\left[C(1) C^{\dagger}(1)\right]^{1 / 4}}\left[C(m) C^{\dagger}(m)\right]^{1 / 4} \eta .
$$

At this point, we note that one can use the following DWF action to reduce the memory consumption in RHMC.

$$
S_{p f}^{N_{f}=1}=\Phi^{\dagger}\left(C_{1}^{\dagger} C_{1}\right)^{1 / 2} \Phi+\phi^{\dagger}\left(C^{\dagger} C\right)^{-1 / 2} \phi
$$


where $\Phi$ and $\phi$ are independent pseudofermion fields. Then the fermion force due to $N_{p}$ poles can be computed in $n$ subsets, each with multiple-shift CG. Thus the memory consumption can be reduced by a factor of $n$. However, it takes more time to compute these $n$ subsets than just one set with $N_{p}$ poles. To save time, one may apply the multiple-time scale method to these $n$ subsets. Nevertheless, one cannot apply the mass preconditioning for this action, which may be a drawback of this approach. In the following, we use (1.1) for RHMC in all tests.

\section{Memory Requirements for EOFA and RHMC}

Defining $M_{S}=L^{3} T \times[8$ bytes (for double precision real number) $]$, then the link variables (with each $S U$ (3) matrix in the format of 2-column storage) take $M_{U}=48 M_{S}$, the conjugate momenta $M_{P}=32 M_{S}$, and a $5 \mathrm{D}$ vector (on the 5-dimensional lattice) $M_{V}=24 N_{s} M_{S}$, where $N_{s}$ is the extent in the fifth dimension.

For EOFA, it takes $2 M_{U}$ to store the old and new gauge configurations, $M_{P}$ for the conjugate momenta, $2 \times 24 M_{S}$ for $\phi_{1}$ and $\phi_{2}$ (pseudofermion fields) of each fermion, and $M_{P}$ for the fermion force. To compute the fermion force by conjugate gradient, it needs $3.5 \times M_{V}$ for the working space. Thus the memory requirement for EOFA (with one heavy mass preconditioner) amounts to

$$
M_{\mathrm{EOFA}}=2 M_{U}+2 M_{P}+2 \times 48 M_{S}+3.5 \times M_{V}=8\left(32+10.5 N_{s}\right) M_{S} .
$$

For RHMC, it takes $2 M_{U}$ (for old and new gauge configurations), $2 M_{P}$ (for conjugate momenta and fermion force), and $2 \times 12 N_{s} M_{S}$ for the pseudofermion fields (the light fermion and the heavy mass preconditioner) after taking into account of even-odd preconditioning. To compute the fermion force, it needs $\left(2+2 N_{p}\right) 5 \mathrm{D}$ vectors for mult-shift CG and working space, where $N_{p}$ is the number of poles used in the rational approximation. Thus the memory requirement for RHMC is

$$
M_{\mathrm{RHMC}}=2 M_{U}+2 M_{P}+\left(3+2 N_{p}\right)\left(24 N_{s} M_{S}\right)=8\left[20+3\left(3+2 N_{p}\right) N_{s}\right] M_{S} .
$$

From (2.1) and (2.2), the ratio is

$$
\frac{M_{\mathrm{RHMC}}}{M_{\mathrm{EOFA}}}=\frac{20+3\left(3+2 N_{p}\right) N_{s}}{32+10.5 N_{s}},
$$

independent of the size of the 4D lattice. For example, for $N_{p}=12$ and $N_{s}=16$, the ratio is 6.58 . In other words, for HMC of one-flavor QCD with DWF on the $32^{3} \times 64 \times 16$ lattice, EOFA takes $12 \mathrm{~GB}$, while RHMC with $N_{p}=12$ requires at least 79 GB. Obviously, the memory-saving feature of EOFA has significant impacts to large-scale simulations on any platforms, especially for GPUs.

\section{Computational Efficiencies of EOFA and RHMC}

To compare the efficiencies of EOFA and RHMC, we perform the following tests:

1. $N_{f}=1 \mathrm{QCD}$ on the $8^{3} \times 16 \times 16$ lattice

(a) Conventional DWF with kernel $H=2 H_{w}\left(2+\gamma_{5} H_{w}\right)^{-1}$ (i.e., $c=1, d=1 / 2$, and $\omega=1$ ) and $m_{0}=1.8$. 


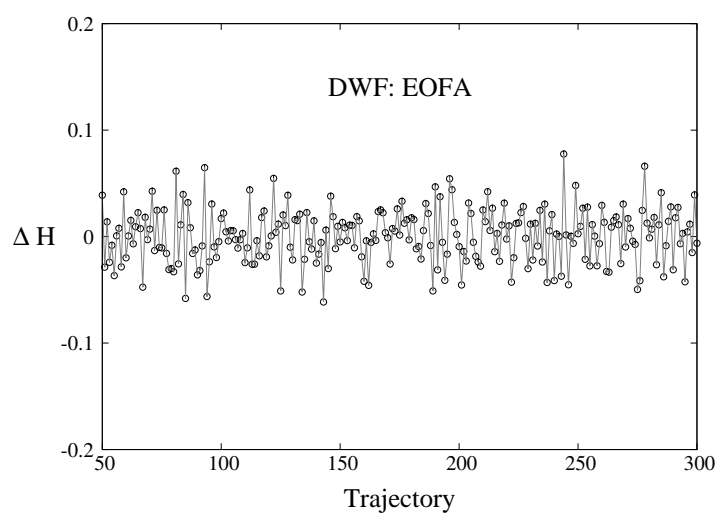

(a)

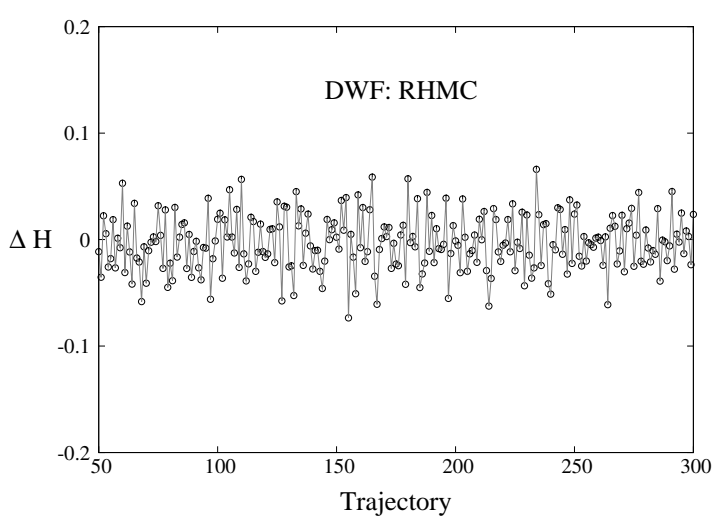

(b)

Figure 1: The change of Hamiltonian $\Delta H$ versus the trajectory in the HMC of one-flavor QCD with the conventional DWF, for (a) EOFA, and (b) RHMC respectively. The line connecting the data points is only for guiding the eyes.

(b) Optimal DWF with kernel $H=H_{w}$ (i.e., $c=1, d=0$, and the reflection-symmetric $\omega$ with $\left.\lambda_{\min } / \lambda_{\max }=0.05 / 6.2\right)$ and $m_{0}=1.3$.

2. $N_{f}=1$ and $N_{f}=(2+1)$ QCD on the $16^{3} \times 32 \times 16$ lattice

(a) Conventional DWF with kernel $H=H_{w}\left(2+\gamma_{5} H_{w}\right)^{-1}$ (i.e., $c=1 / 2, d=1 / 2$, and $\omega=1$ ) and $m_{0}=1.8$.

In all cases, the gauge action is the Wilson plaquette action at $\beta=6 / g^{2}=5.95$. In the molecular dynamics, we use the Omelyan integrator [5], the multiple-time scale method [6], and the auxiliary heavy fermion field [7]. For $N_{f}=1 \mathrm{QCD}$, the sea-quark mass is set to $m_{q} a=0.01$, with the heavy mass preconditioner $m_{H} a=0.1$ for conventional (optimal) DWF. For $N_{f}=(2+1)$ QCD, the sea-quark masses are set to $m_{u / d} a=0.003$ with the heavy mass preconditioner $m_{H} a=0.03$, and the values of $m_{s} a=0.01$ and its mass preconditioner $m_{H_{s}} a=0.1$. In RHMC, the number of poles in the optimal rational approximation of $\left(C C^{\dagger}\right)^{-1 / 2}$ and $\left(C_{1}^{\dagger} C_{1}\right)^{1 / 4}$ is fixed to $N_{p}=12$ for the lattice size $8^{3} \times 16 \times 16$, while $N_{p}=14$ for $16^{3} \times 32 \times 16$.

3.1 $L^{3} \times T=8^{3} \times 16$

First, we compare the HMC chracteristics of EOFA and RHMC. In Fig. 1, we plot the change of Hamiltonian $\Delta H$ versus the trajectory number (after thermalization), for EOFA and RHMC respectively. In both cases, $\Delta H$ is quite smooth without any spikes. Moreover, the measured values of $\langle\exp (-\Delta H)\rangle$ are:

\begin{tabular}{|l||c|r|}
\hline & EOFA & RHMC \\
\hline Conventional DWF & $1.0003(16)$ & $1.0038(17)$ \\
\hline Optimal DWF & $0.9991(17)$ & $0.9994(18)$ \\
\hline
\end{tabular}



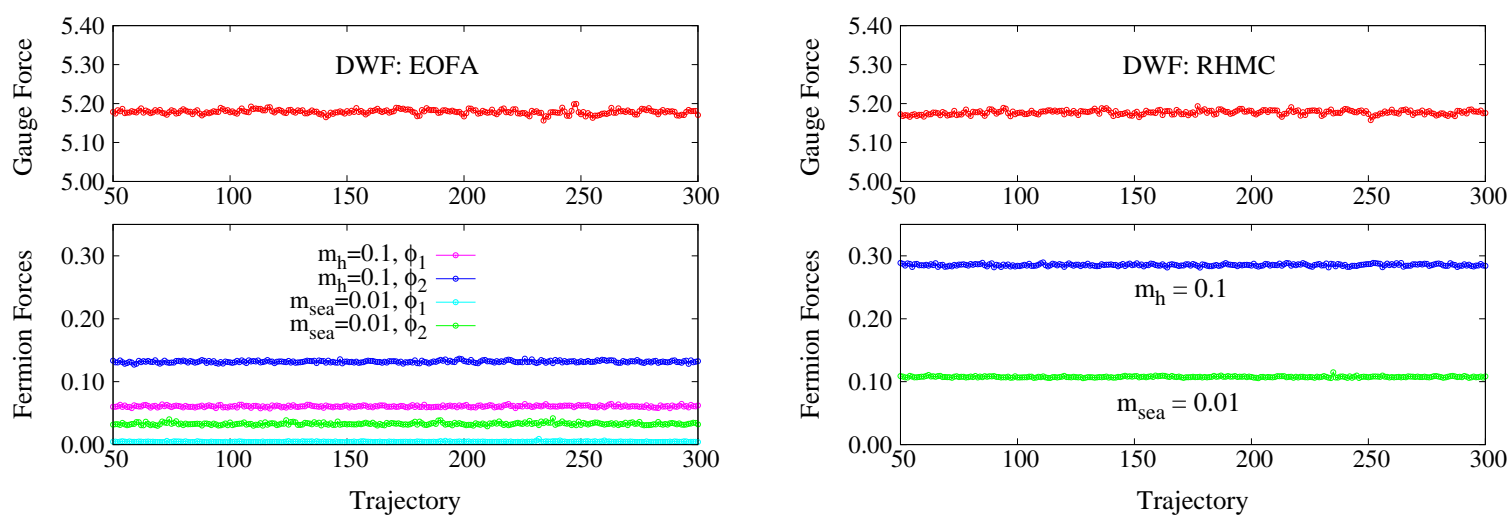

(a)

(b)

Figure 2: The maximum forces of the gauge field and the pseudofermion fields versus the trajectory in the HMC of one-flavor QCD: (a) EOFA, (b) RHMC.

They are all in good agreement with the condition $\langle\exp (-\Delta H)\rangle=1$ which follows from the areapreserving property of HMC.

In Fig. 2, we plot the maximum force (averaged over all links) in each trajectory, for the gauge field, the heavy fermion field, and the light fermion field respectively. For both EOFA and RHMC, the fermion forces behave smoothly in all trajectories. However, the fermion forces of EOFA are substantially smaller than their counterparts in RHMC. The averages of the maximum fermion forces are:

\begin{tabular}{ccccccc}
\hline & \multicolumn{4}{c}{ EOFA } & \multicolumn{2}{c}{ RHMC } \\
& $\left(F_{\phi_{1}}\right)_{\text {light }}$ & $\left(F_{\phi_{2}}\right)_{\text {light }}$ & $\left(F_{\phi_{1}}\right)_{\text {heavy }}$ & $\left(F_{\phi_{2}}\right)_{\text {heavy }}$ & $(F)_{\text {light }}$ & $(F)_{\text {heavy }}$ \\
Conventional DWF & $0.0046(1)$ & $0.0331(2)$ & $0.0609(1)$ & $0.1318(2)$ & $0.1076(1)$ & $0.2855(1)$ \\
\hline Optimal DWF & $0.0009(2)$ & $0.0139(2)$ & $0.0487(1)$ & $0.1810(1)$ & $0.0695(3)$ & $0.3534(1)$ \\
\hline
\end{tabular}

Note that for EOFA, the fermion forces of $\phi_{1}$ are much smaller than their counterparts of $\phi_{2}$. This immediately implies that $\phi_{1}$ and $\phi_{2}$ can be updated at two different time scales. This will be exploited in the tests on the $16^{3} \times 32 \times 16$ lattice.

For tests of $N_{f}=1$ QCD on the $8^{3} \times 16 \times 16$ lattice, we set the multiple-time scales as follows. With the length of the HMC trajectory equal to one, three different time scales are set to $\left\{k_{0}, k_{1}, k_{2}\right\}=\{1,1,10\}$, and the fields are updated according to the following assignment:

$k_{0}: U_{\mu}$ (gauge field),

$k_{1}: \phi_{1}$ (EOFA, heavy fermion), $\phi_{2}$ (EOFA, heavy fermion), $\phi$ (RHMC, heavy fermion),

$k_{2}: \phi_{1}$ (EOFA, light fermion), $\phi_{2}$ (EOFA, light fermion), $\phi$ (RHMC, light fermion). 
Thus the smallest time interval in the molecular dynamic is $1 /\left(k_{0} k_{1} k_{2}\right)$, and the numbers of momentum updates for $\left\{k_{0}, k_{1}, k_{2}\right\}$ are $\left\{8 k_{0} k_{1} k_{2}+1,4 k_{1} k_{2}+1,2 k_{2}+1\right\}$ respectively, according to the Omelyan integrator.

Using one core of Intel i7-3820 CPU@3.60GHz, we measure the average time per HMC trajectory $(\mathrm{T})$ and the acceptance rate $(\mathrm{A})$ after thermalization, and obtain the following results.

\begin{tabular}{ccccc}
\hline & \multicolumn{2}{c}{ EOFA } & \multicolumn{2}{c}{ RHMC } \\
& T (seconds) & A & T (seconds) & A \\
Conventional DWF & $6293(77)$ & $0.980(9)$ & $7365(96)$ & $0.996(4)$ \\
\hline Optimal DWF & $8916(263)$ & $0.980(9)$ & $10657(538)$ & $0.984(8)$ \\
\hline
\end{tabular}

Thus, in both cases (conventional DWF and optimal DWF), EOFA outperforms RHMC for $N_{f}=1$ QCD on the $8^{3} \times 16 \times 16$ lattice.

3.2 $L^{3} \times T=16^{3} \times 32$

Next we turn to tests of $N_{f}=1$ and $N_{f}=(2+1)$ QCD on the $16^{3} \times 32 \times 16$ lattice, for the conventional DWF with kernel $H=H_{w}\left(2+\gamma_{5} H_{w}\right)^{-1}$. The details of the simulation of 2-flavors of DWF have been presented in Ref. [4]. After the initial thermalization of 300 trajectories (done with a GPU), we pick one configuration and use 4 cores CPU of i7-4820K CPU@3.70GHz to continue the HMC simulation with EOFA and RHMC respectively, and accumulate 5 trajectories in each case. With the length of the HMC trajectory equal to one, four different time scales are set to $\left\{k_{0}, k_{1}, k_{2}, k_{3}\right\}=\{10,1,3,2\}$, and the fields are updated according to the following assignment:

$k_{0}: U_{\mu}$ (gauge field),

$k_{1}: \phi_{2}$ (EOFA, heavy fermion), $\phi$ (RHMC, heavy fermion),

$k_{2}: \phi_{1}$ (EOFA, heavy fermion), $\phi_{2}$ (EOFA, light fermion), $\phi$ (RHMC, light fermion),

$k_{3}: \phi_{1}$ (EOFA, light fermion).

Then the smallest time interval in the molecular dynamic is $1 /\left(k_{0} k_{1} k_{2} k_{3}\right)$, and the numbers of momentum updates for $\left\{k_{0}, k_{1}, k_{2}, k_{3}\right\}$ are $\left\{16 k_{0} k_{1} k_{2} k_{3}+1,8 k_{1} k_{2} k_{3}+1,4 k_{2} k_{3}+1,2 k_{3}+1\right\}$ respectively, according to the Omelyan integrator.

With the statistics of five trajectories (all accepted), the average time (seconds) for generating one HMC trajectory (after thermalization) is listed below.

\begin{tabular}{|c|cc|}
\hline & EOFA & RHMC \\
\hline$N_{f}=1$ & $93241(290)$ & $119445(408)$ \\
$N_{f}=2+1$ & $143099(833)$ & $172569(588)$ \\
\hline
\end{tabular}

These results suggest that EOFA outperforms RHMC for $N_{f}=1$ and $N_{f}=(2+1)$ QCD with the conventional DWF. 


\section{Conclusion}

In this paper, we compare the performances of EOFA and RHMC, for $N_{f}=1$ and $N_{f}=2+1$ QCD with DWF, on the $8^{3} \times 16 \times 16$ and $16^{3} \times 32 \times 16$ lattices respectively. Our results suggest that EOFA outperforms RHMC, no matter in terms of the computational efficiency or the memory requirement. This makes EOFA a better choice for dynamical simulations of lattice QCD with DWF. Currently, TWQCD Collaboration is using EOFA to simulate lattice QCD with $(u, d, s, c)$ quarks on the $24^{3} \times 48 \times 16$ and $32^{3} \times 64 \times 16$ lattices, with Nvidia GPUs (GTX-TITAN).

\section{Acknowledgments}

This work is supported in part by the Ministry of Science and Technology (No. NSC102-2112M-002-019-MY3) and NTU-CQSE (Nos. 103R891404).

\section{References}

[1] Y. C. Chen and T. W. Chiu [TWQCD Collaboration], Phys. Lett. B 738, 55 (2014)

[2] M. A. Clark and A. D. Kennedy, Phys. Rev. Lett. 98, 051601 (2007)

[3] T. W. Chiu, Phys. Rev. Lett. 90, 071601 (2003)

[4] T. W. Chiu [TWQCD Collaboration], J. Phys. Conf. Ser. 454, 012044 (2013)

[5] I.P. Omelyan, I.M. Mryglod, and R. Folk, Phys. Rev. Lett. 86, 898 (2001).

[6] J. C. Sexton and D. H. Weingarten, Nucl. Phys. B 380, 665 (1992).

[7] M. Hasenbusch, Phys. Lett. B 519, 177 (2001) 